THE AstrophysiCAL JourNaL, 500:291-301, 1998 June 10

(C) 1998. The American Astronomical Society. All rights reserved. Printed in U.S.A.

\title{
WHERE IS THE DOUGHNUT? LUMINOUS BLUE VARIABLE BUBBLES AND ASPHERICAL FAST WINDS
}

\author{
Adam Frank, ${ }^{1}$ Dongsu Ryu, ${ }^{2,3}$ and Kris Davidson ${ }^{4}$ \\ Received 1997 May 2; accepted 1998 January 16
}

\begin{abstract}
In this paper, we address the issue of the origin of luminous blue variable (LBV) bipolar bubbles. Previous studies have explained the shapes of LBV nebulae, such as $\eta$ Car, by invoking the interaction of an isotropic fast wind with a previously deposited, slow aspherical wind (a "slow torus"). In this paper we focus on the opposite scenario in which an aspherical fast wind expands into a previously deposited isotropic slow wind. Using high-resolution hydrodynamic simulations, which include the effects of radiative cooling, we have completed a series of numerical experiments to test if and how aspherical fast winds effect wind-blown bubble morphologies. Our experiments explore a variety of models for the latitudinal variations of fast wind flow parameters. The simulations demonstrate that aspherical fast winds can produce strongly bipolar outflows. In addition, the properties of outflows recover some important aspects of LBV bubbles that the previous slow torus models cannot.
\end{abstract}

Subject headings: hydrodynamics - ISM: bubbles — stars: mass loss — stars: supergiants

\section{INTRODUCTION}

In just a few years, the Hubble Space Telescope (HST) has transformed our understanding of the massive unstable stars know as luminous blue variables (LBVs). Recent observations have revealed a number of LBVs or LBV candidates to be surrounded by extended aspherical outflows. The most extraordinary of these is the markedly bipolar nebula surrounding $\eta$ Carinae ("the homunculus": Hester et al. 1991; Ebbets et al. 1993; Humphreys \& Davidson 1994). Other LBVs show nebulae with varying degrees of asphericity from elliptical (R127: Nota et al. 1995) to strongly bipolar (which we define though the presence of an equatorial waist) (HR Carinae: Nota et al. 1995; Weis et al. 1996).

These morphologies are quite similar to what has been observed in planetary nebulae (PNs) which arise from lowmass stars (Balick 1987; Manchado et al. 1996). The aspherical shapes of PNs have been successfully explained through a scenario termed the generalized interacting stellar winds model (GISW) (Kwok, Purton, \& Fitzgerald 1978; Kwok 1982; Kahn 1983; Frank et al. 1993; Frank \& Mellema 1994; Mellema \& Frank 1995). In the GISW model, an isotropic fast wind from the central star (a protowhite dwarf) expands into an aspherical (toroidal) slow wind ejected by the star in its previous incarnation as a asymptotic red giant. High densities in the equatorial plane constrain the expansion of the fast wind. The expanding shock which forms quickly assumes an elliptical prolate geometry. If the ratio of mass density between the equator and pole (a parameter we call $q, q_{\rho}=\rho_{e} / \rho_{p}$ ) is high enough, then the elliptical bubble eventually develops a waist and becomes bipolar.

The similarity of PNs and LBV nebulae has led to the suggestion that both families of objects are shaped in

\footnotetext{
${ }^{1}$ Department of Physics and Astronomy, Bausch and Lomb Building, University of Rochester, Rochester, NY 14627-0171; afrank@alethea.pas.rochester.edu.

2 Department of Astronomy, University of Washington, Box 351580, Seattle, WA 98195-1580; ryu@hermes.astro.washington.edu.

${ }^{3}$ Department of Astronomy \& Space Science, Chungnam National University, Daejeon 305-764, Korea.

${ }^{4}$ Department of Astronomy, University of Minnesota, Minneapolis, MN 55455;kd@ast1.spa.umn.edu.
}

similar ways. In Frank, Balick, \& Davidson (1995; hereafter, FBD), a GISW model for $\eta$ Car was presented in which a spherical "outburst" wind expelled during the 1840 outburst expanded into a toroidal "preoutburst" wind. FBD showed that the resulting bipolar outflow could recover both the gross morphology and kinematics of the Homunculus. Nota et al. (1995; hereafter, NLCS) used a similar model for other LBV nebulae, presenting a unified picture of the development of LBV outflows. More recently, Garcia-Segura, Langer, \& Mac Low (1997; hereafter, GLM) presented a model that also relied on the GISW scenario but that changed the order of importance of the winds. The novel aspect of GLM's study was to include the effects of stellar rotation. Using the wind compressed disk model of Bjorkman \& Cassinelli (1992), GLM showed that a strong equator-to-pole density contrast would likely form during the outburst when the star is close to the Eddington limit and rotation can deflect wind streamlines toward the equator. In their model, it is the "postoutburst" mass loss (which was not considered in either FBD or NLCS) that acts as the fast wind. The postoutburst wind in GLM's model "inflates" the bipolar bubble via its interaction with the toroidal outburst wind.

All of these models have demonstrated the potential efficacy of the GISW scenario by recovering the basic shapes observed in LBV nebulae. However, by relying on the presence of a slow torus, they are troubling in their mutual inconsistency. Specifically, the question, Where is the torus? must be answered. Does the torus form during the outburst phase as in GLM, or does it form in the preoutburst wind as in FBD and NLCS? Without invoking binary interactions or a preexisting disk left over from the stellar formation process, it may be difficult to get a strong toroidal density contrast in the preoutburst environment.

Stepping back further, one can also ask if a disk is needed at all. The latter question arises from consideration of new HST images of $\eta$ Car (Morse et al. 1998) that reveal the disk to be so highly fragmented that it may be more reasonable to consider the structure to be a "skirt" of individual clumps of ejecta rather than a continuous feature. This point is crucial, since a discontinuous equatorial spray of isolated bullets cannot hydrodynamically constrain an iso- 
tropic stellar wind to form a bipolar outflow. Thus we are led to consider an alternative model to the one proposed by FBD, NLCS, and GLM which further generalizes the GISW model by turning that scenario on its head. It what follows, we consider the case of an aspherical fast wind interacting with an isotropic slow wind. We imagine a fast wind ejected with higher velocity along the poles than along the equator. The question we wish to answer is can such a wind, expanding into an isotropic environment, account for the shapes of LBV nebulae?

There are a number of reasons for pursuing this line of investigation, some of which were cited above as criticisms of the "classic" GISW model. More importantly, however, theoretical models admit the possibility of aspherical fast winds in massive stars. Pauldrach \& Puls (1990) have shown that a discontinuity (bistability) in mass loss and velocity occurs when the effective gravity of the star drops below a critical value. Lamers \& Pauldrach (1991) used these results to demonstrate that stellar rotation can induce latitudinal changes in $g_{\text {eff }}$ and the optical depth of the wind. The change in optical depth puts the polar and equatorial regions of the star on different sides of the bistabilty limit. A high-velocity, low-density wind forms at the poles, and a low-velocity, high-density wind forms along the equator. It should be noted that the wind-compressed disk (WCD) model of Bjorkman \& Cassinelli (1992) also produces aspherical winds, since the equatorial focusing occurs close to the star. Thus, a wind that has been shaped by the WCD mechanism, if it is expanding into a slower moving environment, should be considered an aspherical fast wind, i.e., the issue is always the velocity (and density) of previously ejected material. It is worth noting, however, that recent numerical models of the WCD mechanism (Owocki, Cranmer, \& Gayley 1996), which include nonradial line forces, found inhibition of the wind compression and mass loss in the equator. Instead, a net flow in the poleward direction was formed. This is, therefore, yet another way by which fast winds might become aspherical. Finally, and most importantly, there is direct evidence for asphericity in fast winds. Observations of the wind of AG Carinae (Leitherer et al. 1994) imply a pattern of densities and velocities from pole to equator much like that described in Lamers \& Pauldrach (1991). Finally, we note that it is worthwhile to pursue this kind of investigation simply because it has not been done before. The GISW model and its variations have been very successful in accounting for a variety of bipolar outflow phenomena (Mellema, Eulderink, \& Icke 1991; Blondin \& Lundqvist 1993; Frank, Balick, \& Livio 1996). Since the effect of aspherical fast winds has yet to be investigated, the potential of finding useful results is high, which argues for a detailed study.

We note that this paper represents an initial exploratory study. We are using an admittedly ad hoc formalism to control the asphericity of the fast wind, and we have not tuned our parameters to the accepted values for any particular LBV. Our purpose in this paper is to map out the broad consequences of including aspherical fast winds into the GISW formalism with an application to LBVs as a class of outflows. In future papers, we will attempt to apply our results to individual LBVs in an attempt to make detailed contact with observational results.

The organization of the paper is as follows. In $\S 2$ we describe the numerical method and initial conditions used in our simulations. In $\S 3$ we present and discuss the results of our simulations. In $\S 4$ we present our conclusions along with a discussion of some issues raised by the simulations.

\section{COMPUTATIONAL METHODS AND INITIAL CONDITIONS}

The gasdynamic interactions we wish to study are governed by the Euler equations with a "sink" term in the energy equation due to radiation losses.

$$
\begin{gathered}
\frac{\partial \rho}{\partial t}+\nabla \cdot \rho \boldsymbol{u}=0, \\
\frac{\partial \rho \boldsymbol{u}}{\partial t}+\nabla \cdot \rho \boldsymbol{u} \boldsymbol{u}=0, \\
\frac{\partial E}{\partial t}+\nabla \cdot \boldsymbol{u}(E+p)=-\frac{\rho}{\bar{m}} \Lambda(T),
\end{gathered}
$$

where

$$
E=\frac{1}{2} \rho|\boldsymbol{u}|^{2}+\frac{1}{(\gamma-1)} p
$$

and

$$
p=\rho \frac{k}{\bar{m}} T .
$$

In the above equations, $\bar{m}$ is the mean mass per particle and $\gamma$ is the ratio of specific heats, which we take to be $\gamma=5 / 3$.

We have carried out our study using the total variation diminishing (TVD) method of Harten (1983) as implemented by Ryu et al. (1995). The TVD code is an explicit method for solving the Euler equations that achieves second-order accuracy by finding approximate solutions to the Riemann problem at grid boundaries while remaining nonoscillatory at shocks through the application of a lower order monotone scheme. For this study, we used the code configured in cylindrical coordinates $(r, z)$.

The cooling term $\Lambda(T)$ was calculated via look-up tables for $\Lambda(T)$ taken from the coronal cooling curve of Dalgarno \& McCray (1972). In the TVD code, the cooling is applied in between hydrodynamic time steps via an integration of the thermal energy $\left(E_{\mathrm{t}}\right)$ equation,

$$
\frac{d E_{t}}{d t}=-\dot{E}_{t}=-\frac{\rho}{\bar{m}} \Lambda(T) .
$$

If the cooling timescale becomes smaller than the dynamical timescale, the cooling term becomes a "stiff" source term. There are several ways to the handle stiff source terms (see, e.g., LeVeque 1997). First, the cooling timescale as well as the Courant condition is considered to determine the time step. Then, in some situations, the time step is governed by the cooing timescale and becomes uncomfortably too small. Second, Strang's operator splitting is employed, where the cooling is computed by multiple steps with its own time step during one hydrodynamic time step. Third, the solution takes the form

$$
E_{t}^{n+1}=E_{t}^{n} \exp \left(-\frac{\dot{E}_{t}^{n}}{E_{t}^{n}} \Delta t\right),
$$

where the superscript $n$ refers to the time index. In this method, the cooling is computed in a single step. It produces good results even though the cooling timescale is 
smaller than the dynamical timescale. When cooling is small, the solution approaches the conventional form

$$
E_{t}^{n+1}=E_{t}^{n}-\dot{E}_{t}^{n} \Delta t .
$$

The third method has been used in our simulations. The term in the exponential can be expressed as $\Delta t / \Delta t_{\mathrm{c}}$, where $\Delta t_{\mathrm{c}}$ is the local cooling time step of the gas, $\Delta t_{c}=\dot{E}_{t}^{n} / E_{t}^{n}$. Although the method works well for small cooling time step, for safety we have used

$$
\Delta t=\min \left[\Delta t_{h}, 1.5 \Delta t_{c}\right],
$$

where $\Delta t_{\mathrm{h}}$ is the dynamical time step set by the Courant condition.

Care must be taken in the application of cooling near contact discontinuities (CDs) wherein typical wind-blown bubble temperatures and densities can vary by more than an order of magnitude going from low to high values of density and high to low values of temperature. In simulations with less-than-perfect resolution, the $\mathrm{CD}$ will be smeared out across a number of cells. In these intermediate zones, which have both high density and high temperature, the cooling term can be anomalously large. This leads to the removal of prodigious amounts of energy from the system at just a few zones. To avoid these problems, we have tracked the $\mathrm{CD}$ by following the advection of a passive tracer $\psi$ via equation (1), i.e.,

$$
\frac{\partial \psi}{\partial t}+\nabla \cdot \psi \boldsymbol{u}=0
$$

This equation has been solved with the TVD code. Using this tracer, we can distinguish between shocks and CDs. If the fast stellar wind has $\psi=1$ and the slow material initially filling the grid has $\psi=0$, then a CD can always be marked as a location with $0.1<\psi<0.9$. When a CD is encountered, we replace the anomalous cooling value with one obtained from an average of the eight nearest neighbor cells that do not contain the CD. Tests of the code show that this formalism allows us to reproduce analytically derived shock speeds (Koo \& McKee 1992) for spherical radiative bubbles in a variety of power-law, $\rho \propto r^{\mathrm{k}}$, environments.

Our numerical experiments are designed to study the evolution of wind-blown bubbles driven by aspherical fast winds. The environment is always assumed to be characteristic of a previously deposited spherically symmetric "preoutburst" wind, which we denote as wind 0 with massloss rate $\dot{M}_{0}$ and velocity $V_{0}$. Thus $\rho_{0}=\dot{M}_{0} / 4 \pi r^{2} V_{0}$. For the driving "fast" or "outburst" wind, which we denote as wind 1 , we need a formalism for setting the latitudinal $(\theta)$ variations in the wind properties, i.e., $\dot{M}_{1}=\dot{M}_{1}(\theta)$ and $V_{1}=V_{1}(\theta)$. We note that since we wish to drive prolate bipolar bubbles, we always assume that the velocity at the poles is larger than at the equator.

We have chosen to explore three models for the pole-toequator variation in wind parameters. Each model is based on the assumption of a different quantity remaining constant across the face of the star. Our three models are

1. Constant momentum input: $\dot{\Pi}=\dot{M}_{1} V_{1}=$ const.

2. Constant energy input: $\dot{E}=\frac{1}{2} \dot{M}_{1} V_{1}^{2}=$ const.

3. Constant density: $\rho_{1}=$ const.

If we choose our fiducial values for the density and velocity at the equator $\left(\rho_{1 e}, V_{1 e}\right)$, then the variation of these quantities can be expressed as $\rho_{1}(\theta)=\rho_{1 e} g(\theta)$ and $V_{1}(\theta)=$ $V_{1 e} f(\theta)$. We can then write $g(\theta) \propto f(\theta)^{n}$. For case 1 where $\dot{\Pi}=$ const $=\dot{\Pi}_{e}$, we have

$$
\begin{aligned}
\dot{\Pi} & =\dot{M}_{1}(\theta) V_{1}(\theta) \\
& =\left[4 \pi R_{*}^{2} \rho_{1}(\theta) V_{1}(\theta)\right] V_{1}(\theta) \\
& =\left(4 \pi R_{*}^{2} \rho_{1 e} V_{1 e}\right) V_{1 e} .
\end{aligned}
$$

Thus,

$$
g(\theta)=\frac{1}{f(\theta)^{2}} .
$$

For case $1, n=-2$. Similarly, for cases 2 and 3, one finds $n=-3$ and 0 , respectively.

For $f(\theta)$, we have chosen an ad hoc function that produces a smooth variation in $V_{1}$ and $\rho_{1}$ from equator to pole. Written in terms of velocity, we have

$$
V_{1}(\theta)=V_{1 e}\left[1-A \frac{\exp \left(-2 B \cos ^{2} \theta\right)-1}{\exp (-2 B)-1}\right]^{-1},
$$

where the constant $B$ determines the shape of the equatorto-pole variability. The constant $A$ is related to the magnitude of the contrast $A=1-q_{\mathrm{v}}$. Note that in what follows, we shall write $q_{\mathrm{x}}=X_{\mathrm{e}} / X_{\mathrm{p}}$, and $X$ is either the velocity or the density. Note that $V_{\mathrm{p}}=V_{\mathrm{e}} /(1-A)$, so $V_{\mathrm{p}}=V_{\mathrm{e}} / q_{\mathrm{v}}$. We take $A<1$, so that together with equation (12) we have $q_{\mathrm{v}}=V_{\mathrm{e}} / V_{\mathrm{p}}<1$ and $q_{\rho}=\rho_{e} / \rho_{p}>1$. These relations express our assumptions about the velocity variation across the face of the star and its consequences for the density distribution given our different models. In Figure 1, we show a plot of the fast wind $V(\theta)$ and $\rho(\theta)$ for each of our three cases.

Using the formalism described above, we have carried out three sets of numerical experiments varying the assumptions about the mass-loss rates in the successive winds between each set. Within the first two sets, we performed a triplet of simulations with the $\theta$ dependence of $\dot{M}_{1}$ and $V_{1}$ corresponding to the three cases discussed above. In set 1 , we examined the interaction between two winds with equal mass-loss rates. These simulations are performed to give us a baseline on the gasdynamic flow pattern. In the second set of experiments, we examined the interaction between two winds in which the previously ejected "slow" preoutburst wind $\left(\dot{M}_{0}\right)$ had a lower mass-loss rate than the outburst wind. We examine this case because stellar evolution models show that the mass-loss rate increases during an LBV eruption (Langer et al. 1994). In the third set of experiments, we examined the interaction of three winds, where $\dot{M}_{0}=\dot{M}_{2}<\dot{M}_{1}$ and $V_{0}<V_{1}<V_{2}$. In these simulations only, the outburst wind is considered to be aspherical. We examine this case to explore the effect of the postoutburst wind on the nebular morphology. We note that the velocity difference between preoutburst and postoutburst winds may not be physically realized. As we shall discuss in $\S 3.3$, this assumption should not effect our results significantly. The initial conditions for each of our nine simulations, including the index $n$ of the density function (i.e., eq. [12]), are given in Table 1.

The mass-loss rates use in our simulations range from $\dot{M}=10^{-6}$ to $\dot{M}=10^{-4} M_{\odot} \mathrm{yr}^{-1}$. Our wind velocities vary between 100 and $1400 \mathrm{~km} \mathrm{~s}^{-1}$. These values are representative of what is observed in LBVs (Leitherer 1997) with the highest velocities seen in $\eta$ Car (Ebbets et al. 1997). We note that in giant outbursts, the mass-loss rates may increase by 

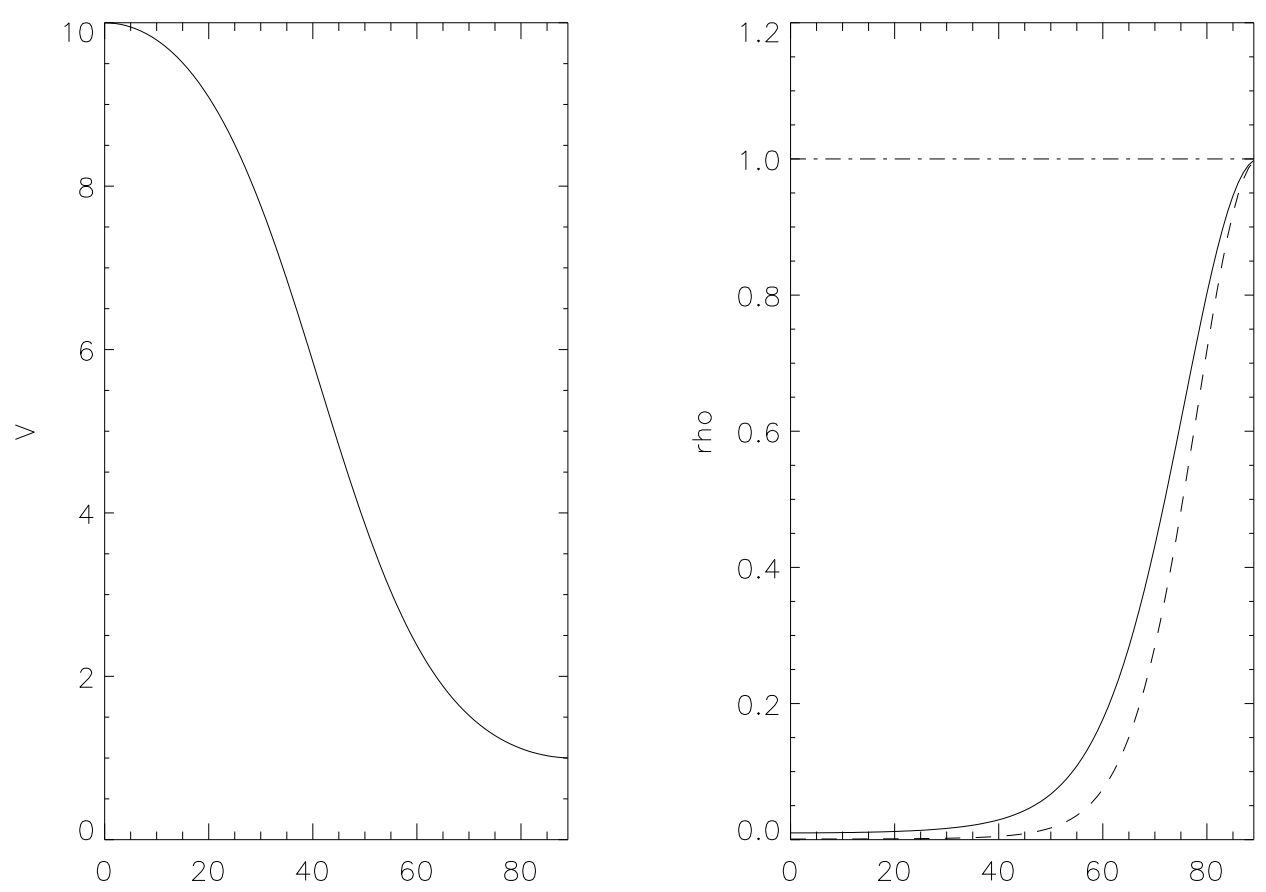

FIG. 1. - Initial conditions for aspherical fast stellar wind. The variation of stellar wind velocity and density with latitude are shown for a model with $A=0.1$ and $B=2$. Note that the equator is at $\theta=90^{\circ}$. The velocity and density at the equator are set to 1 . The density plot shows the three different cases considered in text. Solid line: momentum input $\dot{\Pi}=$ const across face of star. Dashed line: energy input $\dot{E}=$ const. Dash-dotted line: density $\rho=$ const.

an order of magnitude or more over what is used in this study (Humphreys \& Davidson 1994). We have chosen not to use larger values of the mass-loss rate based on the short cooling timescales which occur in high-density winds. Short timescales would force us to use lower resolution simulations given constraints on computational time. We decided that achieving higher resolution $(256 \times 256)$ was a more important goal since, based on our results, we will be able to anticipate the enhanced cooling effects of higher mass-loss rates. We note that the simulations were all run until the outer shock ran off the grid. Thus, the timescale for each simulation is essentially the dynamical age of the bubble for $R \approx 5 \times 10^{17} \mathrm{~cm}$.

\section{RESULTS}

\subsection{Two-Wind Models with Equal $\dot{M}$}

Figure 2 shows $\rho, T, V$, and $P$ for run A after $t=800 \mathrm{yr}$ of evolution. Run A has an outburst stellar wind whose momentum flux, $\dot{\Pi}$, is constant across the face of the star. A number of features of the simulation are noteworthy, but it is first worthwhile to identify the main features of the windblown bubble. The density map shows a bright rim which corresponds to the shell of swept-up preoutburst wind material. It is important to distinguish the composition of the material in the shell (i.e., which wind it originates from) since its high density will make it most luminous and, therefore, the defining feature in a real nebula. Figure 2 shows that the shell develops significant asphericity due to the aspherical driving force of the outburst wind. For later comparison, we introduce a quantity we call the ellipticity, defined as the ratio of the distance from the star to the outer shock in the equator and the pole, $e=R_{\mathrm{e}} / R_{\mathrm{p}} ; e \approx 0.68$ in this simulation. Notice also that the bubble has developed a "waist," which is the observational signature of a bipolar rather than an elliptical configuration. The density map for run $A$ is also shown in Figure 4 with the appropriate reflections to create a slice of the full bubble.

Detailed inspection of the $\rho$ and $T$ maps show that the shell is thin and cold $\left(T \approx 10^{4} \mathrm{~K}\right)$ at all latitudes. The narrowness of the shell is due to the enhanced compression that accompanies radiative losses behind the outer shock. The role of cooling is important and is determined by the relation between the age of the bubble and the cooling timescale. Given a shock speed $V_{\mathrm{s}}$ and a preshock density of $\rho_{\text {pre }}$, the cooling timescale can be defined as $t_{\mathrm{c}}=C V^{3}{ }_{s} / \rho_{\mathrm{pre}}$,

TABLE 1

INITIAL CONDITIONS FOR RUNS A-H

\begin{tabular}{ccccccccc}
\hline \hline Run & $\dot{M}_{0}$ & $V_{0}$ & $\dot{M}_{1}$ & $V_{1}$ & $\dot{M}_{2}$ & $V_{2}$ & $q_{\mathrm{v}}$ & $\begin{array}{c}\text { Condition } \\
\text { Index }\end{array}$ \\
\hline $\mathrm{A} \ldots \ldots$ & $1 \times 10^{-4}$ & 100 & $1 \times 10^{-4}$ & 150 & NA & NA & 0.2 & -2 \\
$\mathrm{~B} \ldots \ldots$ & $1 \times 10^{-4}$ & 100 & $1 \times 10^{-4}$ & 150 & NA & NA & 0.2 & -3 \\
$\mathrm{C} \ldots \ldots$ & $1 \times 10^{-4}$ & 100 & $1 \times 10^{-4}$ & 150 & NA & NA & 0.2 & 0 \\
$\mathrm{D} \ldots \ldots$. & $1 \times 10^{-6}$ & 100 & $1 \times 10^{-4}$ & 150 & NA & NA & 0.2 & -2 \\
$\mathrm{E} \ldots \ldots$. & $1 \times 10^{-6}$ & 100 & $1 \times 10^{-4}$ & 150 & NA & NA & 0.2 & -3 \\
$\mathrm{~F} \ldots \ldots$. & $1 \times 10^{-6}$ & 100 & $1 \times 10^{-4}$ & 150 & NA & NA & 0.2 & 0 \\
$\mathrm{G} \ldots \ldots$ & $1 \times 10^{-6}$ & 100 & $1 \times 10^{-4}$ & 150 & $1 \times 10^{-6}$ & 1400 & 0.3 & -2 \\
$\mathrm{H} \ldots \ldots$ & $1 \times 10^{-6}$ & 100 & $1 \times 10^{-4}$ & 150 & $1 \times 10^{-6}$ & 1400 & 0.14 & -2 \\
$\mathrm{I} \ldots \ldots$. & $1 \times 10^{-6}$ & 100 & $1 \times 10^{-4}$ & 150 & $1 \times 10^{-6}$ & 1400 & 0.1 & -2 \\
\hline
\end{tabular}



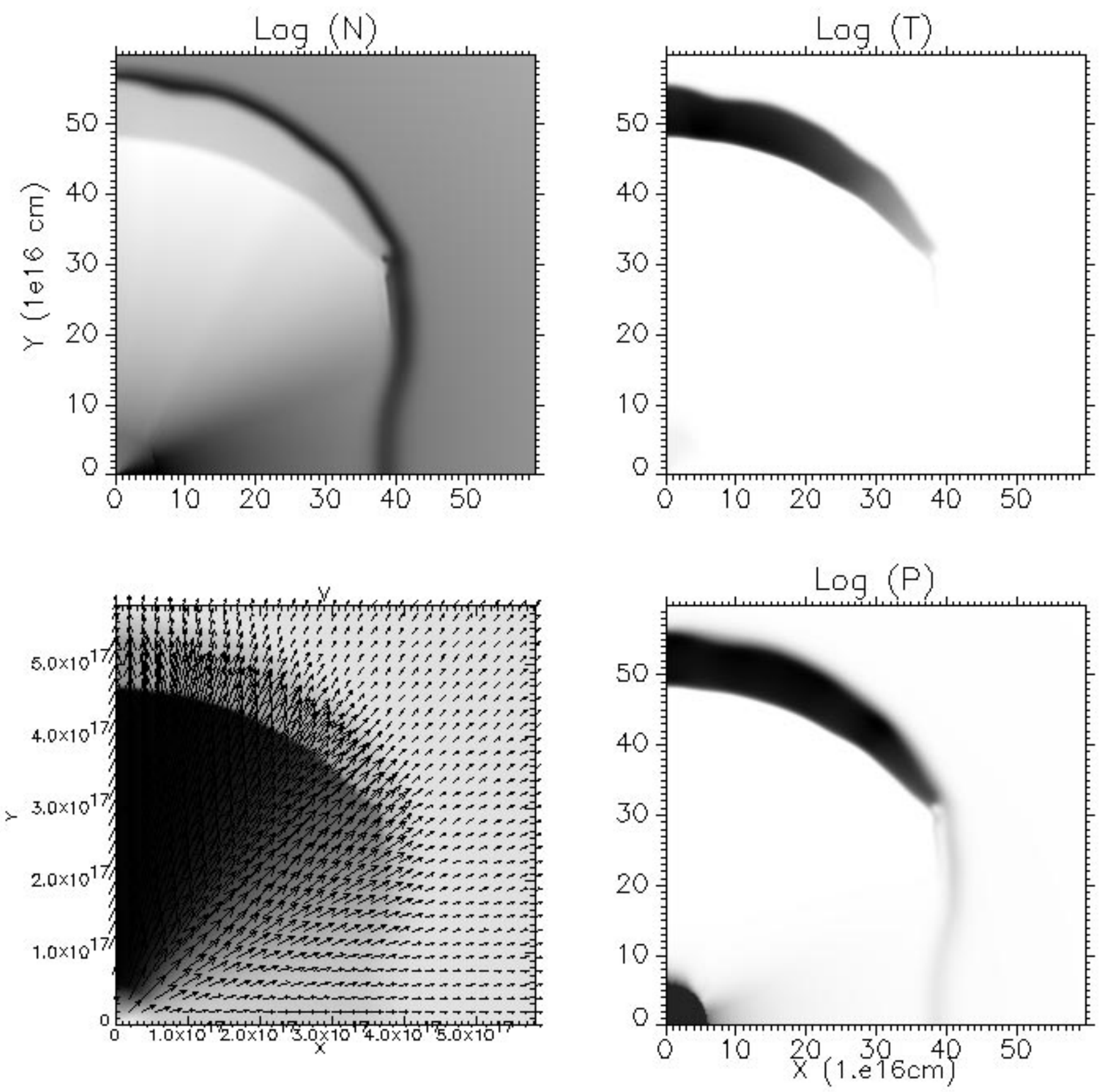

FIG. 2.-Run A after $800 \mathrm{yr}$. Shown are gray-scale maps of $\log _{10}$ number density, temperature, velocity (with vectors superposed), and pressure. Note that dark scales correspond to high values of the variables. The range of variables are as follows: $0.5<\log _{10}\left(n / \mathrm{cm}^{-3}\right)<3.7,3.9<\log { }_{10}(T / \mathrm{K})<6.8,6.1<\log _{10}$ $\left(V / \mathrm{cm} \mathrm{s}^{-1}\right)<7.8$, and $-11.3<\log _{10}\left(P /\right.$ dynes $\left.\mathrm{cm}^{-2}\right)<-7.8$.

where $C=6 \times 10^{-35} \mathrm{~g} \mathrm{~cm}^{-6} \mathrm{~s}^{4}$ (Kahn 1976). The outer shock speed in this simulation is on the order of $200 \mathrm{~km} \mathrm{~s}^{-1}$. Taking $\rho_{\text {pre }}$ from the mass conservation in the preoutburst wind gives $t_{\mathrm{c}}=150 y$, which is short compared to the age of the bubble in Figure 2.

The situation is quite different behind the "reverse" or "inner" shock. At low latitudes, the speed of the outburst wind is relatively small $\left(150 \mathrm{~km} \mathrm{~s}^{-1}\right)$ and the density is relatively high, producing a short cooling time. The reverse situation occurs at the poles, where the high wind velocity $\left(750 \mathrm{~km} \mathrm{~s}^{-1}\right)$ and low preshock density yield cooling times that are of the same order or longer than the age of the bubble. This produces a cap of high-temperature gas at the poles. High thermal pressure enhances the preferential expansion at the poles, which leads to a bipolar bubble. We note, however, that even when the cooling at the poles is strong enough to drain away all shock-thermalized energy, the bubble that is produced is still bipolar.

The density maps for runs $\mathrm{B}$ and $\mathrm{C}$ are presented in Figure 4. Run B corresponds to the case where the energy input in the wind, $\dot{E}_{1}$, is constant across the face of the star. The morphology of the bubble in run B is similar to that in run $A$, the principle difference being the aspect ratio of the bubble. This can be attributed to the differing latitudinal density profiles for the two runs. From equation (12) the $\dot{\Pi}_{1}=$ const simulation (run A) has $\rho \propto f(\theta)^{-2}$. The $\dot{E}_{1}=$ const simulation (run B) yields $\rho \propto f(\theta)^{-3}$. For run C, the density in the wind is constant and is set by the value in the 
equator at which $V_{1}(\theta)$ has its minimum. Since $\rho \propto \dot{M} / V$ via the continuity equation, the outburst wind density in run $\mathrm{C}$ is almost as high as that in the preoutburst wind. This produces a strong ram pressure flux ratio between the two flows. The initial shape of the outburst wind is strongly imprinted on the developing bubble. Thus run $\mathrm{C}$ produces most bipolar shell of all three simulations examined in this first set of experiments.

The results presented above show that bipolar bubbles can form when driven by an aspherical fast wind. The differences between the three models also demonstrate that the degree of bipolarity does depend on relative densities between the aspherical fast (outburst) wind and the spherically symmetric slow preoutburst wind.

\subsection{Two-Wind Models with $\dot{M}_{1}>\dot{M}_{0}$}

As Langer et al. (1994) have demonstrated with stellar evolution codes, the LBV outburst phase is likely to involve an increase in mass loss over the preoutburst wind. Thus we have the case of a "heavy" wind expanding into a light one. In this situation, the preexisting circumstellar material cannot constrain the outflow. The wind will not be significantly decelerated by the environment until the swept-up mass is comparable to the mass ejected by the wind. Given that the outburst mass-loss rate can be an order of magnitude or more greater than the preoutburst value, the outburst wind can expand almost ballistically for some time. If, in addition, the outburst wind is aspherical, then the bubble that is created will retain much of the asphericity imprinted on the wind at the stellar surface. In this scenario, we expect that one gets out something similar to what is put in via the initial density and velocity distributions for the outburst wind. To test this expectation, we have run a second set of experiments consisting of three simulations. Runs D, E, and F have $\dot{\Pi}_{1}, \dot{E}_{1}$, and $\rho_{1}$ held constant, respectively, just as in the simulations described in the last section. In these simulations, however, $\dot{M}_{1}=100 \dot{M}_{0}$. Note that for the sake of consistency with the previous experiments, we have used the same velocity in the preoutburst wind as was used in runs A through C. The results of Langer et al. (1994) show that the velocity in the preoutburst wind should be higher than that in the outburst wind. We do not believe that such a difference will produce a profound change in our results. A highvelocity preoutburst wind will interact with the ISM, producing shocked gas which backflows to the boundary of the preoutburst wind. Such a high-pressure region will still yield an isotropic force opposing the expansion of the outburst wind. Thus, while the details of the flow may change, the global properties most likely will not be affected.

Figure 3 shows a snapshot of the evolution of run D after $t=240 \mathrm{yr}$. As expected, the bubble that is formed appears strongly bipolar with an ellipticity of $e=0.4$. While such a result was expected from intuitive arguments, a ballistic expansion model does not tell the whole story. Note first that run $\mathrm{D}$ has reached the same size $\left(R_{\mathrm{e}}=5.1 \times 10^{17} \mathrm{~cm}\right)$ as run $\mathrm{A}$ in about one-third of the time, giving an expansion velocity along the pole of $V_{\mathrm{s}} \approx 660 \mathrm{~km} \mathrm{~s}^{-1}$. This is less than the outburst wind velocity at the poles, $V_{1}=750 \mathrm{~km}^{-1}$. Ambient material that passes through the shock can be expected to reach high temperatures of the order of $T \sim 10^{7}$ $\mathrm{K}$, where cooling is relatively ineffective. The temperature map clearly shows high- $T$ gas at the top of the bubble. Consideration of the passive tracer shows that this is ambient material that has been accelerated. The tem- perature map also shows lower temperature gas at smaller radii. This is outburst wind material that has passed through the inner shock which can be seen at $R_{\mathrm{e}}=4.2$ $\times 10^{17} \mathrm{~cm}$. Consideration of the position of the shock compared with the position expected for ballistic winds yields a inner shock velocity of $V_{\mathrm{s}} \approx 200 \mathrm{~km} \mathrm{~s}^{-1}$, which is consistent with the temperature in this region (measured to $T \approx 10^{6}$ $\mathrm{K})$. The presence of this shock indicates that the outburst wind gas parcels are being decelerated by the swept-up ambient material. This deceleration occurs because of the low outburst wind density along the poles as can see from the density map. Recall that we are using equator-to-pole velocity contrast of $q_{\mathrm{v}}=1 / 5$. This produces a equator-topole density contrast of $q_{\rho}=1 / q_{v}^{2}=25$.

Runs $\mathrm{E}$ and $\mathrm{F}$ show similar morphologies, though the $\rho=$ constant case again produces the most bipolar configuration. We note that for run $\mathrm{E}$ and $\mathrm{F}, e=0.42$ and 0.36 , respectively. In Figure 4 we present $\log _{10} \rho$ maps for runs $A$ through $\mathrm{F}$. In each map, we have reflected the computational space about the symmetry axis $(r=0)$ and symmetry plane $(z=0)$ to show the full bubble cross section and facilitate comparison. In spite of the differences between the runs, Figure 4 demonstrates the principle conclusion of our first two sets of experiments: an aspherical stellar wind can drive an aspherical bubble.

\subsection{Three-Wind Models}

In this section, we present the results of our final set of numerical experiments in which we take a step closer to reality by invoking three-wind models. In runs $\mathrm{G}, \mathrm{H}$, and $\mathrm{I}$, we have performed simulations with conditions in the preoutburst and outburst winds similar to run D. In these runs, however, the outburst wind lasts only 30 years, after which a "postoutburst" wind is driven into the grid. The characteristics of the postoutburst wind were meant to mimic the conditions currently observed in $\eta$ Carinae in the sense that we used a relatively low mass rate and high velocity for the wind, i.e., $\dot{M}_{2}<\dot{M}_{1}$ and $V_{2}>V_{1}$. Recall that in the simulations of GLM, it was the fast postoutburst wind that was responsible for driving the bipolar $\eta$ Car bubble. In these simulations, we are interested in what effect this postoutburst wind will have on a bipolar bubble created by a previously ejected dense aspherical wind. We note that the postoutburst wind in all of these simulations is spherical and will produce an isotropic driving force.

We have run three simulations, each of which utilized the $\dot{\Pi}=$ const formalism. In these simulations it was $q_{\mathrm{v}}$, the equator-to-pole velocity contrast in the outburst wind, that was varied. The purpose of this strategy was to understand how the additional driving force provided by the postoutburst wind would effect bubbles with different degrees of bipolarity.

Figure 5 shows a snapshot of the evolution of run $\mathrm{H}$ after $t=250 \mathrm{yr}$. This simulation has an outburst wind with $q_{\mathrm{v}}=$ $1 / 7$ and $q_{\rho}=49$. Unlike the last set of experiments, here the gas ejected during the outburst in these models occupies a thin shell. The reasons for this are twofold. First, the outburst had a short intrinsic lifetime (which we denote as $t_{1}$ ), so its intrinsic width is $\delta R=V_{1} t_{1}$. Second, the outburst material has been decelerated and compressed from the outside by the preoutburst wind and compressed and accelerated from the inside by the action of the succeeding postoutburst wind. Note that the postoutburst wind has itself been decelerated through the action of a strong inner shock. 

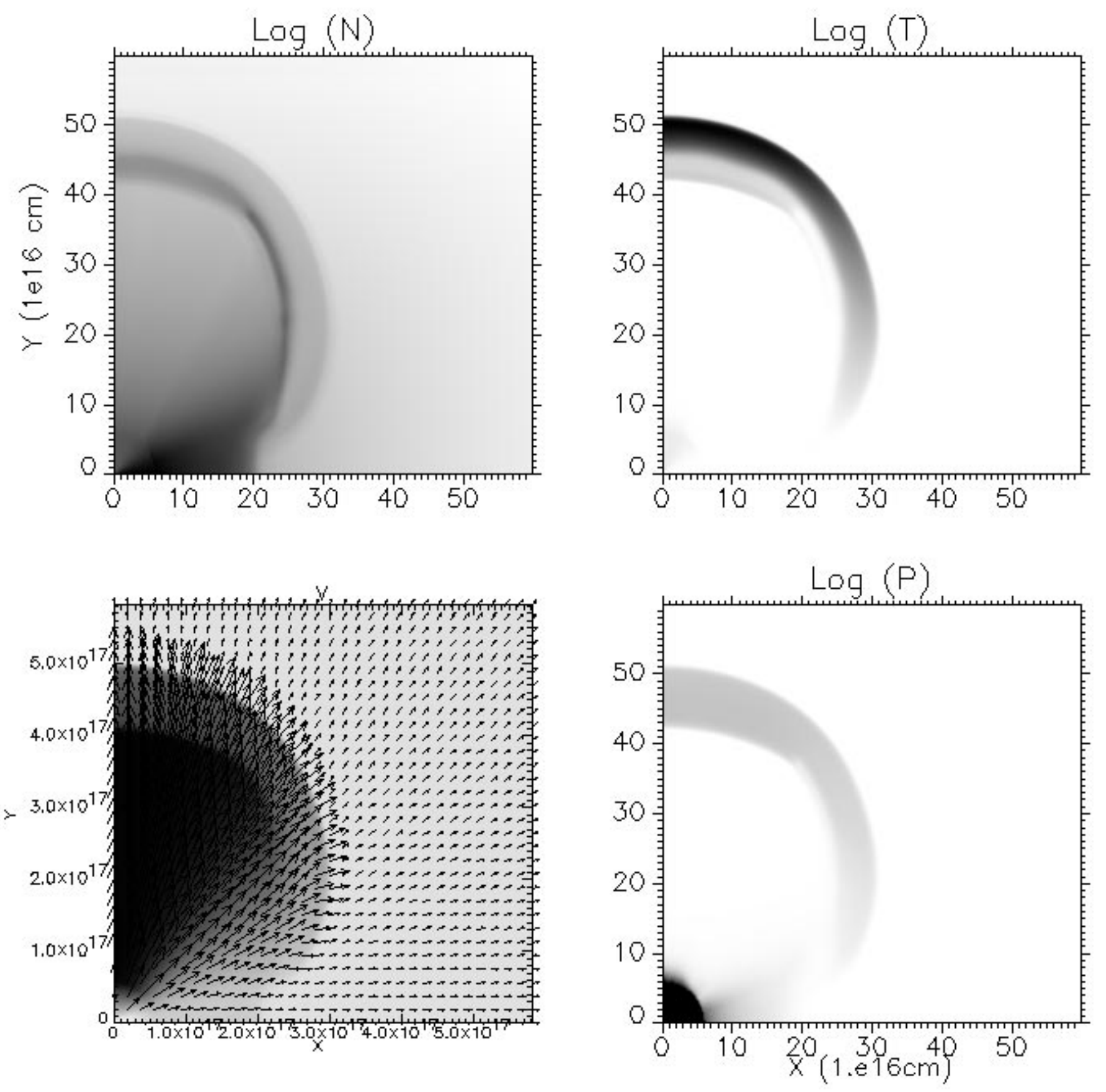

Fig. 3.-Run D after 240 yr. The maps and parameter ranges are the same as in Fig. 2.

The temperature behind this shock at the poles is quite high $\left(T \approx 2 \times 10^{7} \mathrm{~K}\right)$, reflecting the high initial velocity of the postoutburst wind. The inner shock has a highly aspherical configuration. The radially streaming postoutburst wind encounters this shock at oblique angles and is refracted toward the symmetry axis (Frank \& Mellema 1996). This produces strong shearing flows in the region behind the inner shock which appear to give rise to instabilities in the interface separating the shocked postoutburst and outburst flows.

In Figure 6 we present $\log _{10} \rho$ maps for all three simulations from this set of experiments. Again it is clear that strong bipolar morphologies develop without the need for a slow-moving disk. Comparison between the simulations shows that decreasing $q_{\mathrm{v}}$ produces stronger bipolar mor- phologies. It is worth noting that if the expansion of the bubble were ballistic, then we would expect $e \approx q_{\mathrm{v}}$, since $R_{\mathrm{e}}=V_{1 e} t$ and $R_{\mathrm{p}}=V_{1 p} t$. This is not the case. Thus, these bubbles show the result of significant hydrodynamic shaping. Some part of the change in the shape results from the deceleration of the outburst wind via the previously ejected material. The postoutburst wind, however, also contributes by accelerating the outburst wind material. As the bubble evolves, this acceleration will have its greatest effect near the equator, where the outburst wind has been most strongly decelerated. Thus, the action of the postoutburst wind will be to drive the bubble toward a more spherical configuration as system evolves. We note, however, that the postoutburst wind might also be aspherical. As was noted above, Leitherer et al. (1994) have observed two com- 

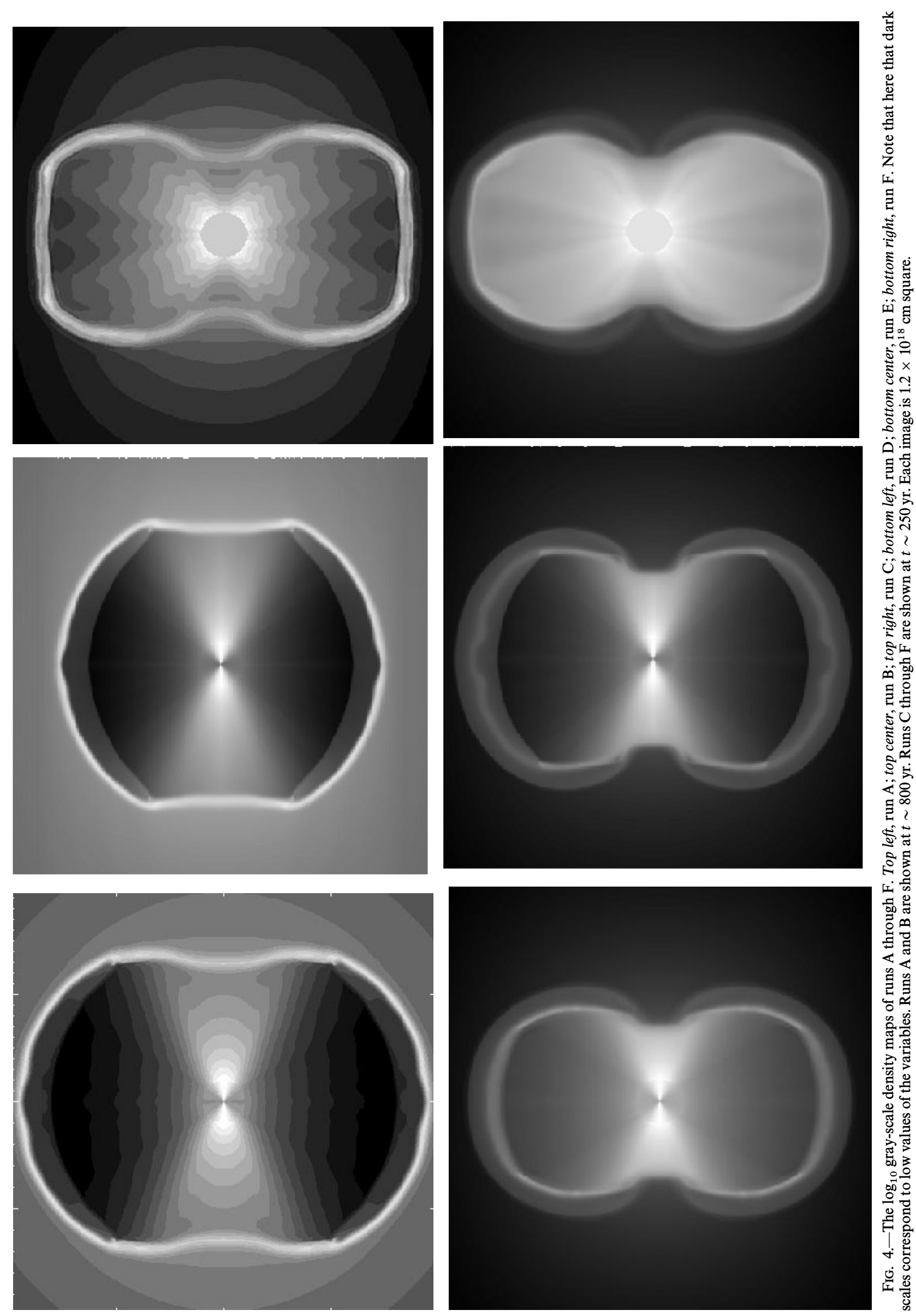

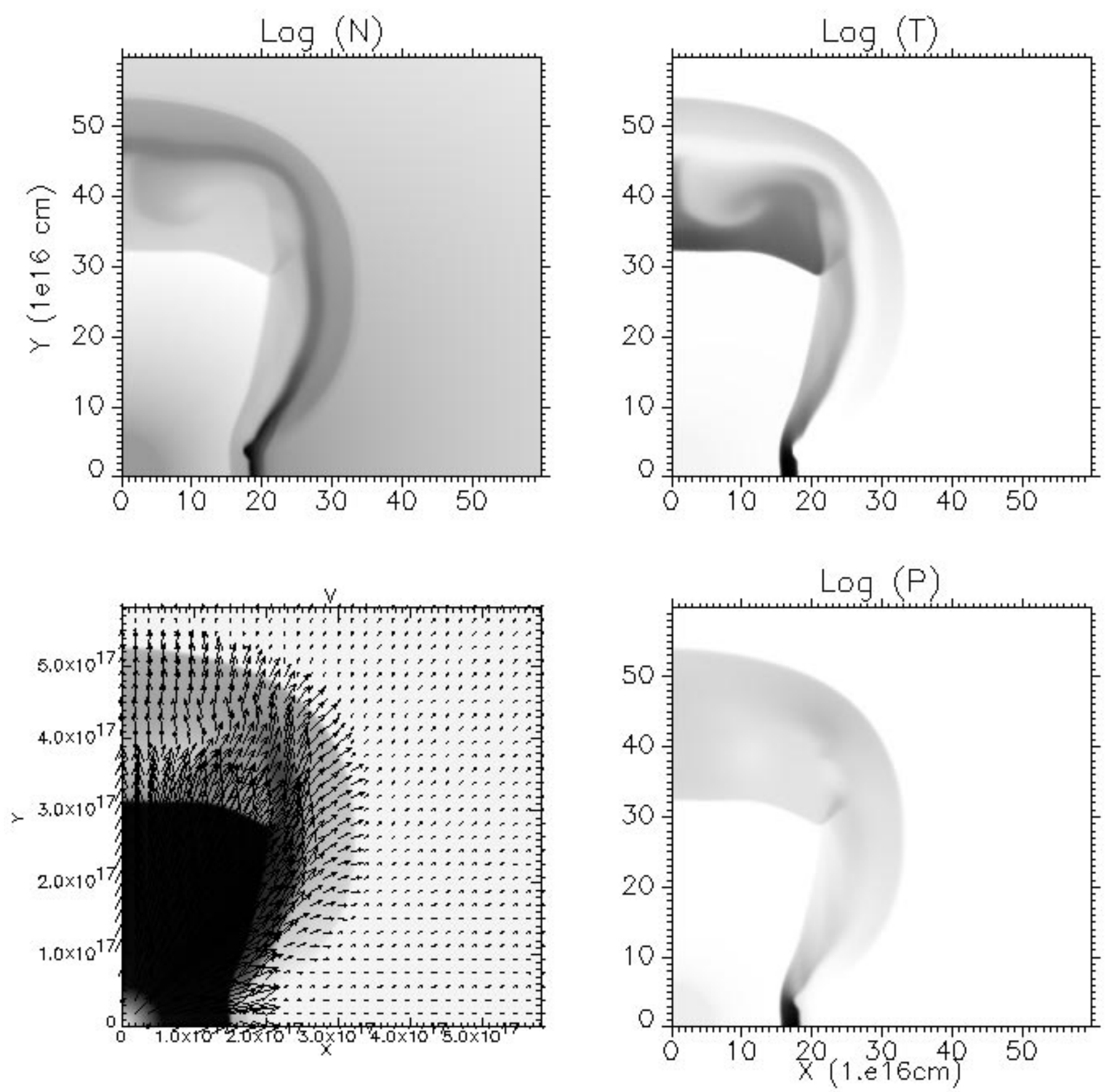

Fig. 5.-As in Fig. 2, but for run G after $250 \mathrm{yr}$. The range of variables are as follows: $0.5<\log _{10}\left(n / \mathrm{cm}^{-3}\right)<3.1,3.9<\log _{10}(T / \mathrm{K})<7.5,6.5<\log _{10}$ $\left(V / \mathrm{cm} \mathrm{s}^{-1}\right)<8.1$, and $-11.3<\log _{10}\left(P /\right.$ dynes $\left.\mathrm{cm}^{-2}\right)<-7.7$.

ponents in the wind of AG Car. A strong aspherical postoutburst wind would then increase the elongation of the bipolar lobes. Further observational study of the characteristics of LBV winds are warranted to determine the latitudinal density and velocity variations.

\section{DISCUSSION AND CONCLUSIONS}

The results of our simulations demonstrate that bipolar wind-blown bubbles can result purely from the action of an aspherical fast wind. In previous studies of LBV bubbles (FBD; NLCS; GLM), it has been assumed that a slowmoving torus or disk of gas was a necessary precondition for the development a bipolar bubble. Our results indicate that the properties of LBV bubbles may not require such a torus to form either before (FBD; NLCS) or during (GLM) the outburst.

Our scenario has a number of attractive features. First, it is both observationally and theoretically motivated. From the observational side, there is already evidence that LBV winds can take on aspherical velocity and density distributions. From the theoretical side, the theory of Lamers \& Pauldrach (1991) have demonstrated that " bistable" winds are possible around massive hot stars. In addition, the diversity of shapes of LBV bubbles (NLCS) may be difficult to achieve with preexiting disk models. The models presented here can recover the diversity of shapes simply by chang- 


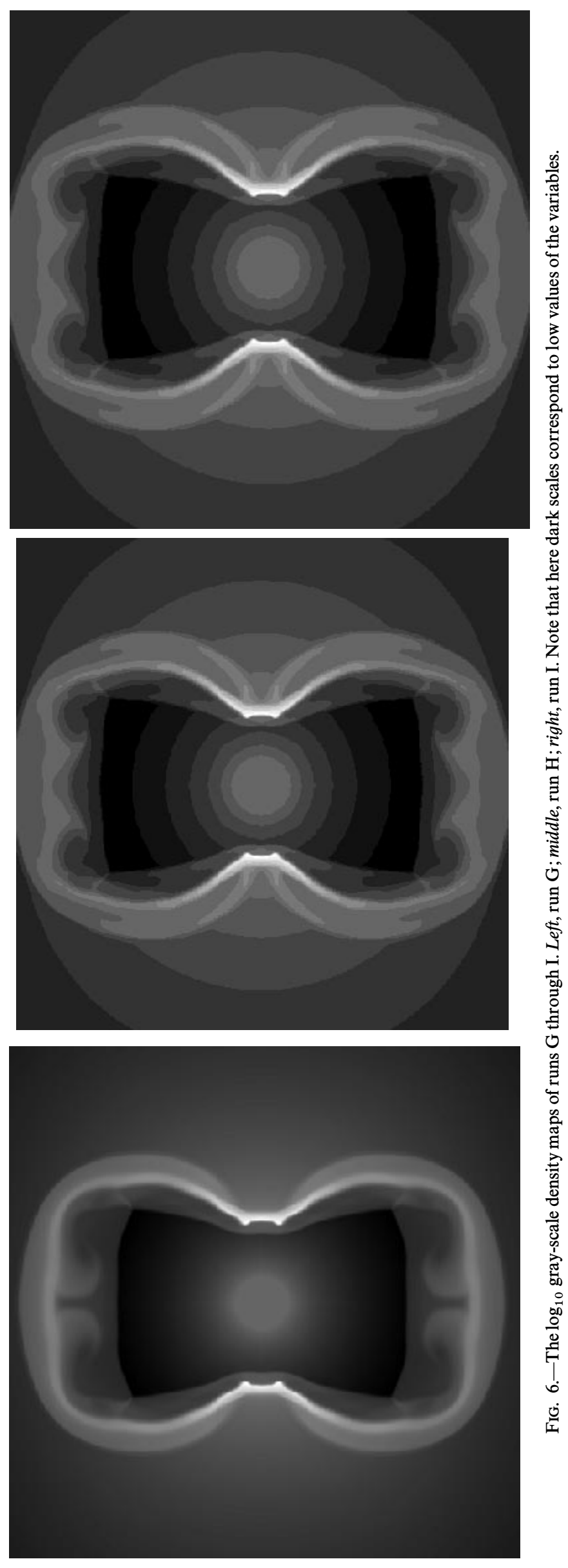


ing $q_{\mathrm{v}}$, although it is certainly true that this also begs the question of what drives the velocity contrast in the fast wind.

Currently, it is unclear what form the mass distribution takes in the lobes of LBV bubbles. One critical test of the different models for LBV nebula shaping would entail comparison of latitudinal variations of mass. If the caps of a bipolar lobe have densities that are comparable to that in the lobe's flanks, it would present difficulties for the slow torus models. In a bipolar bubble resulting from a spherical fast wind driving into a slow torus, the caps of the bubble should be the location of the lowest density. For aspherical fast winds, however, high or equal density in the poles poses no significant problem, since we are then seeing a signature of the latitudinal dependence of the fast wind density (consider runs $\mathrm{C}$ and $\mathrm{F}$ in Fig. 3). We wish to note also that Currie et al. (1996a) and Morse et al. (1998) have found that the shape of $\eta$ Car is best matched by a geometry that can be described as a "double flask" rather than an two osculating spheres. Based on comparison of published results, the models presented here seem to do a better job of recovering such a shape than either FBD, NLCS, or GLM.

It is noteworthy, however, that the scenario presented here would not produce the equatorial skirt seen surrounding the waist of the homunculus in $\eta$ Car. The presence of that feature is what motivated the original application of the GISW slow torus models. Within the current formulation of the aspherical fast wind model there is not a likely means of producing such a feature. A few points are worth noting, however. The equatorial skirt is not a continuous or even axisymmetric feature. Thus, whatever its origin, it is hard to imagine that it can be the agent that constricts a spherical fast wind and produces the bipolar bubble. In addition, there are a number of "spikes" extending beyond, but connecting with, the homunculus that have been reported to have velocities in excess of $1000 \mathrm{~km}^{-1}$ (Meaburn et al. 1998). They yield dynamical timescales $\leq t_{0}$, where $t_{0}$ is the time since the outburst (Currie et al. 1996b). Thus it is possible that the equatorial skirt is actually a spray of material that was ejected at some point after the outburst of 1849 and that was decelerated by its passage through the dense shell of the outburst wind. The nonaxisymmetric distribution of the skirt may then be a consequence of the intrinsic pattern of ejecta or of impulsive instabilities that will occur when the ejecta is driven through the outburst wind.

Regardless of the answer to this issue, the results presented here show that there are two very different scenarios for the formation of LBV bubbles. Either they form via the interaction of a spherical fast wind driving into an aspherical slow wind (a slow torus), or they form from an aspherical fast wind driving into an isotropic preexisting environment. This embarrassment of riches can be eventually be dealt with by comparing the latitudinal distributions of mass and momentum observed in real bipolar LBVs with what is predicted for the various models. Such an approach was used successfully by Masson \& Chernin (1992) in evaluating different models of molecular outflow formation in YSOs. This project is currently in progress.

We wish to thank Jon Morse, Jon Bjorkman, Tom Jones, and Stan Owocki for the very useful and enlightening discussions on this topic. Support for A. F. was provided by NASA grant HS-01070.01-94A from the Space Telescope Science Institute, which is operated by AURA Inc. under NASA contract NASA-26555. Additional support for A. F. came from NSF Grant AST-9702484 and the Minnesota Supercomputer Institute. Partial support for D. R. was provided from Seoam Scholarship Foundation.
Balick, B. 1987, AJ, 94, 671

Bjorkman, J., \& Cassinelli, J. 1992, ApJ, 409, 429

Blondin, J., \& Lundqvist, P. 1993, ApJ, 405, 337

Currie, D., Dowling, D., Shaya, E., \& Hester, J. 1996a, in The Role of Dust in Star Formation, ed. H. Kaufl \& R. Siebenmorgen (New York: Springer), 89

Currie, D., Dowling, D., Shaya, E., Hester, J., Scowen, P., Groth, E., Lynds, R., \& O'Neil E. 1996b, AJ, 112, 1115

Dalgarno, A., \& McCray, R. 1972, ARA\&A, 10, 375

Ebbets, D., Garner, H., White, R., Davidson, K., \& Walborn, N. 1993, in Circumstellar Media in the Late Stages of Stellar Evolution, ed. R. E. S. Clegg, I. R. Stevens, \& W. P. X. Meikle (Cambridge: Cambridge Univ. Press)

Ebbets, D., Morse, J., Davidson, K., \& Walborn, N. 1997, in ASP Conf. Proc. 120, Luminious Blue Variables: Massive Stars in Transition, ed. A. Nota \& J. G. L. M. Lamers (San Francisco: ASP), 249

Frank, A., Balick, B., \& Davidson K. 1994, ApJ, 441, L77 (FBD)

Frank, A., Balick, B., Icke, V., \& Mellema, G. 1993, ApJ, 404, L25

Frank, A., Balick, B., \& Livio, M. 1996, ApJ, 471, L53

Frank, A., \& Mellema, G. 1994, ApJ, 430, 800 1996, ApJ, 472, 684

Garcia-Segura, G., Langer, N., \& Mac Low, M. 1997, in ASP Conf. Proc. 120, Luminious Blue Variables: Massive Stars in Transition ed. A. Nota \& J. G. L. M. Lamers, (San Francisco: ASP), 332

Harten, A. 1983, J. Comp. Phys., 49, 357

Hester, J. J., Light, R. M., Westphal, J. A., Currie, D. G., \& Groth, E. J. 1991, AJ, 102, 654

Humphreys, R., \& Davidson, K. 1994, PASP, 106, 1025

Kahn, F. 1976, A\&A, 50, 145

Kahn, F. D. 1983, in IÁU Symp. 103: Planetary Nebulae, ed. D. Flower (Dordrecht: Reidel)

\section{REFERENCES}

Koo, B., \& McKee, C. F. 1992, ApJ, 388, 103

Kwok, S. 1982, ApJ, 258, 280

Kwok, S., Purton, X., \& Fitzgerald, X. 1978, ApJ, 219, L125

Lamers, H., \& Pauldrach, A. 1991, A\&A, 244, 5L

Langer, N., Hamann, W.-R., Lennon, M., Najarro, F., Pauldrach, A., \& Puls, J. 1994, A\&A, 290, 819

Leitherer, C. 1997, in ASP Conf. Proc. 120, Luminious Blue Variables: Massive Stars in Transition, ed. A. Nota \& J. G. L. M. Lamers (San Francisco: ASP), 58

Leitherer, C., et al. 1994, ApJ, 428, 292

LeVeque, R. J. 1997, in Computational Methods in Astrophysical Fluid Flows (Berlin: Springer), in press

Manchado, A., Guerrero, M., Stanghelli, L., \& Serra-Ricart, M. 1996, The IAC Morphological Catalog of Northern Galactic Planetary Nebulae (La Laguna: IAC)

Masson, C. R., \& Chernin, L. M. 1992, ApJ, 387, L47

Meaburn, J., Boumis, P., Walsh, J. R., Steffan, W., Holloway, A. J., Williams, R. J. R., \& Bryce, M. 1998, MNRAS, in press

Mellema, G., Eulderink, F., \& Icke, V. 1991, A\&A, 252, 718.

Mellema, G., \& Frank, A. 1995, MNRAS, 273, 401

Morse, J., Davidson, K., Ebbets, M., \& Walborn, N., Balick, B., Frank, A., \& Bally, J. 1998, AJ, submitted

Nota, A., Livio, M., Clampin, M., \& Schulte-Ladbeck, R. 1995, ApJ, 448, 788 (NLCS)

Owocki, S., Cranmer, S., \& Gayley, K. G. 1996, ApJ, 472, L115

Pauldrach, W. A., \& Puls, J. 1990, A\&A, 237, 409

Ryu, D., Brown, G. L., Ostriker, J. P., \& Loeb, A. 1995, ApJ, 452, 364

Weis, K., Duschl, W., Bomans, D., Chu, Y.-H., \& Joner, M. 1996, A\&A, in press 